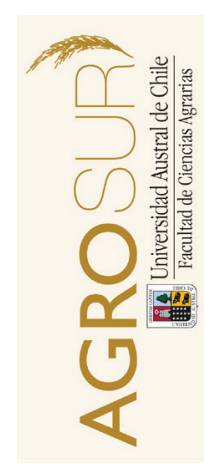

\section{¿Qué nos quiere decir el Suelo?}

\author{
What does the Soil wants to tell us?
}

\author{
Zúñiga, $\mathrm{F}^{a, b^{*}}$, Dörner, J. ${ }^{a, b}$, Thiers, O. $^{b, c}$, Blum, W.E.H. ${ }^{d}$ \\ ${ }^{a}$ Instituto de Ingeniería Agraria y Suelos, Facultad de Ciencias Agrarias, \\ Universidad Austral de Chile. Casilla 567, Valdivia, Chile. \\ ${ }^{b}$ Centro de Investigación en Suelos Volcánicos, Universidad Austral de Chile. Casilla 567, Valdivia, Chile. \\ 'Instituto de Bosques y Sociedad, Facultad de Ciencias Forestales, \\ Universidad Austral de Chile. Casilla 567, Valdivia, Chile. \\ ${ }^{d}$ University of Natural Resources and Life Sciences (BOKU). Peter-Jordan-Str. 82, 1190 Vienna, Austria.
}

\begin{abstract}
A B S T R A C T
This explicit point of view explains several soil functions, including the least known function of soil as an intergenerational living archive. Well-known scientific approaches are related to their features and functions, in particular to the influence of human activity. However, the importance of productive, environmental and cultural historical heritage sites does not seem to be sufficiently clear and investigated. In the context of global change this function is particularly important, both locally and globally, supporting the management of the sustainable use of land and soil (productive, social and environmental). According to the worldwide proposal of the United Nations, we recommend to nominate the $5^{\text {th }}$ of December as the National Day of the Soil.
\end{abstract}

Viewpoints

Soil Science

${ }^{*}$ Corresponding author:

Felipe Zúñiga

E-mail address:

felipezunigaugalde@gmail.com

\title{
RESUMEN
}

Este punto de vista, expresado de manera sucinta y directa, recuerda una de las varias funciones del suelo, quizás la menos conocida, referida a la función de ser un archivo histórico vivo intergeneracional. Conocidos son los enfoques eruditos referidos a sus características y funciones, en especial, las productivas relacionadas con el hombre. Sin embargo, la función de reserva histórica productiva, ambiental y cultural de la humanidad, aparece como menos conocida y estudiada. Actualmente en el contexto del cambio global, precisamente dicha función cobra especial relevancia, tanto a nivel local como global, como aporte base para gestionar su uso sustentable (productivo, social y ambiental). Por lo tanto, en sintonía con lo propuesto a nivel mundial, donde el suelo posee una consideración explícita por parte de la Naciones Unidas, nos atrevemos a proponer la celebración del 05 de diciembre, de cada año, como el Día Nacional del Suelo.

\section{La visión humana del Suelo}

El suelo ha sido definido como la interacción entre el clima, material parental, topografía, organismos en función del tiempo y, todo lo anterior, bajo la influencia en mayor o menor medida de la humanidad (Jenny, 1941). Por lo tanto, podemos asegurar que como humanidad hemos co-evolucionado con el suelo, donde ambos hemos experimentado, directa o indirectamente, el rigor de los procesos de formación; en nuestro caso específico, esta experiencia ha forjado nuestro carácter como especie, permitiendo mantenernos vigentes en el tiempo. Los más eruditos en este tema, los pedólogos y edafólogos, han definido el concepto de pedón, el cual según Bockheim et al. (2005), surgió como una interrogante: ¿Cuál es la unidad más pequeña que puede ser reconocida en el paisaje como un "suelo"?. De acuerdo a la definición planteada por R.W. Simonson, D.R. Gardner y W.M. Johnson en la década de los 60's en los EE.UU., "el pedón fue identificado como la unidad tridimensional (volumen) más pequeña de suelo que muestra todos los horizontes presentes y sus relaciones". Nosotros como científicos, con una visión de funcionalidad, lo hemos retratado de manera diferente (Kübiena, 1950; Hartemink, 2009); buscado comprender su funcionamiento, estudiando sus características, propiedades y procesos (Simonson, 1959), y con ello, hacer uso de el, por medio de su modificación o transformación, para suplir nuestras necesidades alimentarias (Ramankutty y Foley, 1999); incluso relevando algunas de sus características y propiedades como alternativas de mitigación al cambio climático (Lal, 2004). 


\section{El Suelo como "un ser pensante"}

Todas estas definiciones y actividades las hemos realizado de acuerdo a las necesidades que como sociedad hemos requerido a lo largo de nuestra historia, donde el recurso suelo ha sido usado de acuerdo con nuestros propios criterios, creencias y herencias culturales. No obstante, si nos imagináramos al suelo como un "ser pensante", podrían surgir una serie de interrogantes, tales como: ¿quiere el suelo ser definido?, ¿quiere el suelo ser retratado?, ¿quiere el suelo ser usado como nuestro sostén de alimentos?, ¿quiere ser propuesto como una solución a los problemas derivados de nuestro accionar?, y finalmente ¿qué nos quiere decir el suelo en realidad? Y es, en esta interrogante, donde cobra fuerza la función del suelo como archivo de la historia.

Gran parte de nuestra historia como humanidad se basa en lo que podemos encontrar en el suelo, en lo que él nos ha querido preservar y también en lo que aún no nos quiere mostrar. Haciendo un símil tecnológico, el suelo es nuestro disco duro, donde se guarda información a diferentes niveles, tanto de rápido como lento acceso, e incluso, siendo capaz de archivar información parcial e incompleta. Cisternas et al. (2005) y Atwater et al. (2013), nos muestran suelos enterrados y su relación con la historia sísmica de parte del Sur de Chile, asociada principalmente a los tsunamis provocados por mega eventos sísmicos (Figura 1a).

Por otro lado Tuross y Dillehay (1995) dan evidencias de que a través de un mecanismo de preservación orgánica asociado a una capa de turba suprayacente y a un sustrato rico en sílice, se preservaron restos arqueológicos de la cultura Monte Verde que datan de aproximadamente 14.600 años. Mieth y Bork (2005), nos presentan la historia de la erosión de suelo y su efecto social en la cultura Rapa Nui, manifestándose hoy como un agro-ecosistema degradado (Figura 1b).

A un nivel temporal menor, pero espacialmente destacable, el efecto del cambio de uso del suelo ha sido intenso, principalmente a través de la utilización del fuego como herramienta de habilitación y conversión de sistemas naturales a sistemas agrícolas, quedando también su uso registrado en los perfiles de suelo (Figura 1c). Las cicatrices de este proceso son aún identificables, la presencia de una estrata de arcilla calcinada (10 cm de espesor) evidencia esta forma de habilitación para establecer cultivos de trigo en la década del 30'.

\section{Reflexiones respecto a la importancia global del Suelo}

Entonces, de acuerdo a la idea planteada en este punto de vista, pareciera ser que el suelo no quiere sólo ser definido, tampoco busca sólo tener las funciones asignadas por la humanidad y lo que en realidad desea, es ser también un espejo, un portal intergeneracional, donde podemos evidenciar el pasado, que nos muestra lo que fuimos y lo que hicimos como humanidad. Sin embargo, en la sociedad actual pareciera ser que la habilidad contemplativa, la simple descripción, interpretación o sensaciones que provoca el estar frente a un perfil de suelo e intentar descifrar lo que él quiere transmitir, se nubla y en ocasiones se vicia porque buscamos el componente funcional, incluso parcialmente orientado, de esa mirada particular. Es así como es inevitable que razonemos científicamente sobre en cuántos quintales métricos por hectárea (qqm ha ${ }^{-1}$ ) podemos obtener de este suelo, cuántos animales o árboles por hectárea puede soportar, o cuánta biomasa puede llegar a producir, o cuán profundo es para la exploración radical, o de cuántos poros de agua aprovechable dispone, o cuál es su capacidad de uso, y finalmente, cuántos impuestos debemos pagar por él, entre otras muchas preguntas.

Pensamos que quizás -y tan sólo quizás- nos olvidamos de nuestra real capacidad de observación, de las sensaciones que provoca estar frente a frente con el suelo, de la capacidad de dejarse sorprender y quizás desenfocamos nuestra mirada a lo que el suelo nos quiere mostrar. ¿Por qué?, tal vez porque tenemos el temor de ver algo que no nos guste, porque el suelo afortunadamente- no miente y no tiene tapujos en decirnos descarnadamente cómo fuimos, cómo somos y también lo que podemos llegar a ser. Es así como de lo anterior podemos sacar lecciones con historia, las cuales deberíamos considerar en la construcción de nuestro futuro. Tenemos la convicción entonces que el suelo que no quiere ser definido, no quiere ser medido y no quiere ser catalogado. Quizás, tan sólo simplemente quiere ser un espejo y reflejar el espíritu de nuestra imperfecta naturaleza humana desde sus inicios.

En vista de todas las importantes funciones -ecológicas, ingenieriles, culturales y otras que aún no descubrimos- que cumple el suelo para la sociedad humana y el ambiente, en Diciembre del año 2013 las Naciones Unidas en Nueva York han declarado el día 5 de Diciembre como "el Día Mundial del Suelo" y el año 2015 como "el Año Internacional del Suelo". Esto pone de manifiesto que la comunidad internacional comienza a reconocer la importancia de los suelos, lo que va a estimular la enseñanza, la valorización del recurso y sus servicios ecosistémicos, como así también, la relevancia de investigación y educación en suelos en el futuro. Por lo tanto, y siendo consecuente con los principios que inspiran este punto de vista, se propone celebrar desde este año en adelante, en Chile el día 5 de Diciembre como el "Día Nacional del Suelo".

\section{AGRADECIMIENTOS}

Agradecemos a Jasper Moernaut y Mary Negrón por facilitar material gráfico de suelos enterrados en la localidad de Maullín y de praderas en condiciones de degradación en Isla de Pascua (Rapa Nui), respectivamente. 

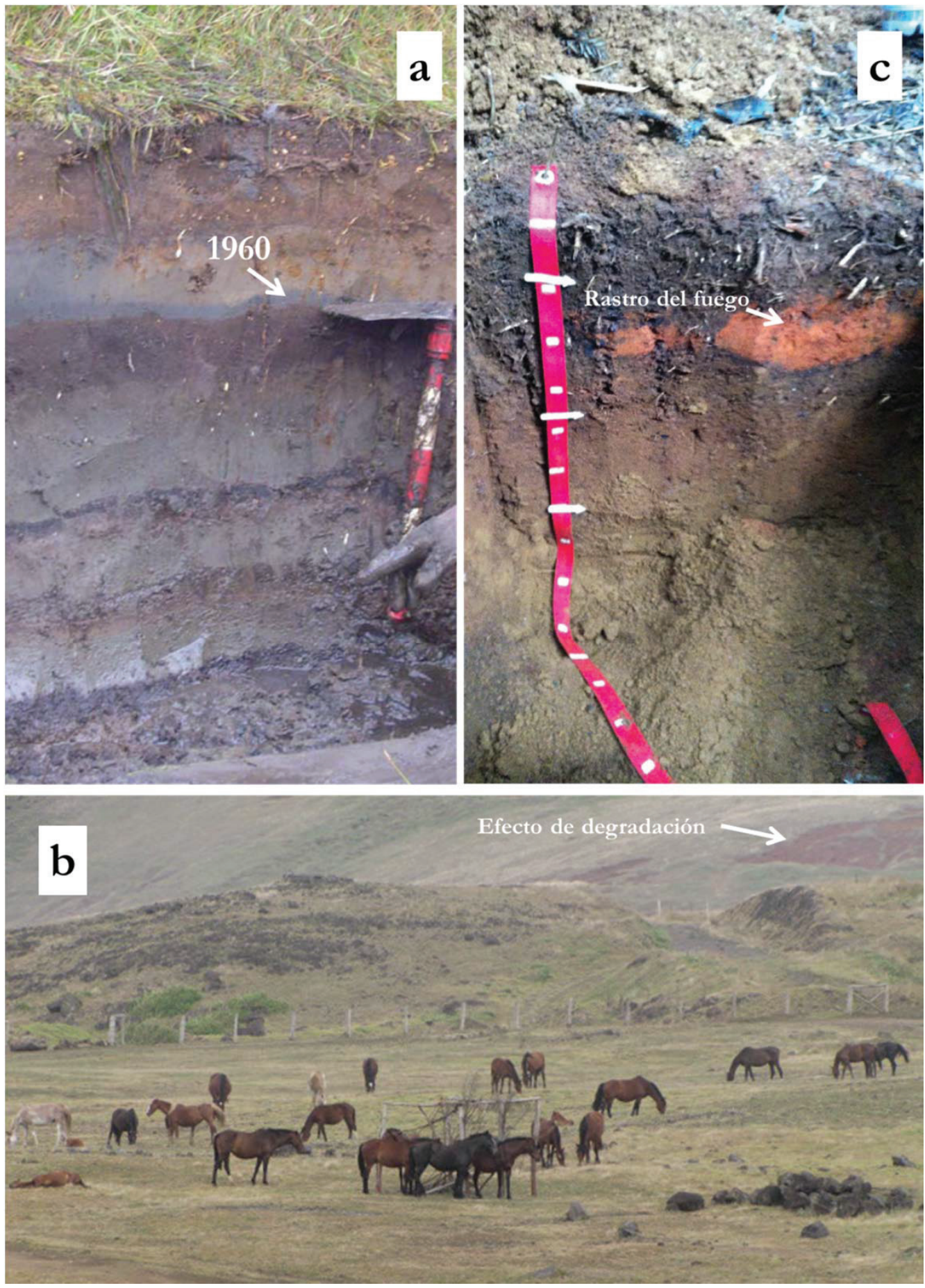

Figura 1. (a) Perfil de suelos enterrados en Maullín por efecto del terremoto de 1960 en Chile. La flecha indica presencia de una estrata de arena depositada por tsunami. (b) Praderas en condiciones de degradación en Isla de Pascua (Rapa Nui). La flecha indica una cárcava en desarrollo. (c) Evidencia del uso del fuego bajo un suelo de matorral de Chusquea quila en los alrededores de la ciudad de Valdivia (Serie de suelos Correltúe).

Figure 1. (a) Profile of buried soil in Maullín as a result of the 1960 Chilean Earthquake. The arrow indicates the presence of a sand stratum deposited by tsunami. (b) Grassland degradation in Easter Island. The arrow indicates a gully development. (c) Evidence of fire use under a Chusquea quila shrub soil near the city of Valdivia (Soil Series Correltúe). 


\section{REFERENCIAS}

ATWATER, B.F., CISTERNAS, M., YULIANTO, E., PRENDERGAST, A.L., JANKAEW, K., EIPERT, A.A., STARIN, W.I., TEJAKUSUMA, I., SCHIAPPACASSE, I., SAWAI, Y. 2013. The 1960 tsunami on beach-ridge plains near Maullín, Chile: Landward descent, renewed breaches, aggraded fans, multiple predecessors. Andean Geology 40(3): 393-418.

BOCKHEIM, J.G., GENNADIYEV, A.N., HAMMER, R.D., TANDARICH, J.P. 2005. Historical development of key concepts in pedology. Geoderma 124: 23-36.

CISTERNAS, M., ATWATER, B.F., TORREJÓN, F., SAWAI, Y., MACHUCA, G., LAGOS, M., EIPERT, A., YOULTON, C., SALGADO, I., KAMATAKI, T., SHISHIKURA, M., RAJENDRAN, C.P., MALIK, J.K., RIZAL, Y., HUSNI, M. 2005. Predecessors of the giant 1960 Chile earthquake. Nature 437: 404-407.

HARTEMINK, A. 2009. The depiction of soil profiles since the late 1700s. Catena 79: 113-127.

JENNY, H. 1941. Factors of Soil Formation. A System of Quan- titative Pedology. Dover, New York.

KÜBIENA, W. 1950. Bestimmungsbuch und Systematik der Böden Europas. Ferdinand Enke Verlag, Stuttgart.

LAL, R. 2004. Soil carbon sequestration to mitigate climate change. Geoderma 123: 1-22.

MIETH, A., BROK, H.R. 2005. History, origin and extent of soil erosion on Easter Island (Rapa Nui). Catena 63: 244260

RAMANKUTTY, N., FOLEY, J.A. 1999. Estimating historical changes in global land cover: Croplands from 1700 to 1992. Global Biogeochemical Cycles 13(4): 997-1027.

SIMONSON, R. 1959. Outlines of a Generalized Theory of Soil Genesis. Soil Science Society Proceedings 23:152-156.

TUROSS, N., DILLEHAY, T.D. 1995. The mechanism of organic preservation at Monte Verde, Chile, and one use of biomolecules in archaeological interpretation. Journal of Field Archaeology 22: 97-110. 